\title{
Integrating Model-Based and Data-Driven Simulator for Health Management of a Reusable Rocket Engine
}

\author{
Daiwa Satoh ${ }^{1}$, Noriyasu Omata ${ }^{1}$, Miki Hirabayashi ${ }^{1}$, Seiji Tsutsumi ${ }^{1}$, Kaname Kawatsu $^{2}$, and Masaharu Abe ${ }^{3}$ \\ ${ }^{1}$ Research Unit III, Research and Development Directorate, Japan Aerospace Exploration Agency (JAXA), \\ Sagamihara, Kanagawa, 252-5210, Japan \\ satoh.daiwa@jaxa.jp \\ omata.noriyasu@jaxa.jp \\ m_hirabayashi@office.so-net.ne.jp \\ tsutsumi.seiji@jaxa.jp \\ ${ }^{2}$ Research Unit III, Research and Development Directorate, Japan Aerospace Exploration Agency (JAXA), \\ Tsukuba, Ibaraki, 305-8505, Japan \\ kawatsu.kaname@jaxa.jp \\ ${ }^{3}$ Aerospace Engineering Solution Division, Business Headquarters, Ryoyu Systems Co., Ltd., \\ Nagoya, Aichi, 455-0024, Japan \\ abe.masaharu@jaxa.jp
}

\begin{abstract}
There is considerable need to generate large amounts of training data that include various operating conditions for fault detection and diagnosis with machine learning in a reusable rocket engine. A system-level simulation model has been developed in which reduced-order models are employed to simulate the global behavior of a reusable rocket engine. Although some components of the engine are not modeled at the system level due to their complexity, they are included among the items inspected during fault detection and diagnosis. This study has developed a regression model for simulating the behavior of such components based on the results of static-firing tests on a reusable rocket engine developed in Japan. The regression model used measurements that can be modeled by the system-level simulation and treated the ones that cannot be modeled as response variables. To identify the operating conditions, the explanatory variables are divided using the Gaussian mixture model in advance, and the Ridge regression models are then trained from the clustered explanatory variables on each cluster. This method reasonably predicts the response variables, even if the static-firing testing includes varying operating conditions such as the combustion phase with varied throttling and the chill-down phase.
\end{abstract}

Daiwa Satoh et al. This is an open-access article distributed under the terms of the Creative Commons Attribution 3.0 United States License, which permits unrestricted use, distribution, and reproduction in any medium, provided the original author and source are credited.

\section{INTRODUCTION}

Many countries have developed reusable launch vehicles to cut launch costs. Although reusable launch vehicles need regular maintenance to maintain reliability, maintenance accounts for most of the launch cost. Four approaches can improve maintenance cost-cutting: a design methodology with long service life as a major consideration; a technique for evaluating remaining component lifetime; nondestructive inspection technology; and fault detection and diagnostics. The authors focus on fault detection and diagnosis in this paper. Reusable launch vehicles need more launches to be operated more effectively. However, since rocket engines are complex and many sensors are needed to monitor the condition of the entire system, manual inspection is time consuming. Further, fault detection and diagnosis of a rocket engine must be finished quickly, so quick and reliable maintenance using only manual inspection has become no longer possible.

To solve the issue, the Japan Aerospace Exploration Agency (JAXA) has developed techniques for fault detection and diagnosis (Kawatsu, 2019; Tsutsumi et al., 2019) for the Reusable Sounding Rocket (RSR), which was developed in Japan (Nonaka et al., 2011; Sato et al., 2014; Kimura et al., 2016). The techniques require vast amounts of training data. Moreover, it is difficult to the anticipate launch conditions because of changing weather and flight conditions. Staticfiring tests take a lot of time and are expensive, so preparing training data using only static-firing tests is also unrealistic. 
The authors have developed a System-Level Simulation (SLS) model to generate training data instead of relying on static-firing tests (Sato et al., 2019). The SLS model simulates the global behavior of an RSR engine by employing reduced-order models. The simulation results in steady-state are highly accurate and obtained by parameter estimation with data assimilation. By changing valve sequences and the pressure and temperature of the tanks, the SLS model can generate time series data under a variety of operating conditions. Many model-based approaches for various products in diverse fields have already been studied (Belapurkar et al., 2011; Frank et al., 2016; Zolghadri et al., 2016; Poon et al., 2017; Sobie et al., 2018; Chowdhury et al., 2019). These studies modeled their targets numerically and used their models for fault detection and diagnosis, but the scope of the studies was limited to behaviors that can only be modeled by the simulation model. In engines, however, there are many parts affected by unknown, complicated physical phenomena. The behavior of sensors installed in such parts cannot be simulated by the SLS model. Half of the sensors used for fault detection and diagnosis measure such physical phenomena. This study suggests a hybrid method that complements the training data with a regression model using sensors that can be modeled by the SLS as explanatory variables and using those that cannot be modeled as response variables.

\section{OVERVIEW OF THE RSR ENGINE}

\subsection{Specification and Operating Conditions}

Figures 1 and 2 are a static-firing test and a system diagram of the RSR engine. An expander bleed cycle is employed in the RSR engine, and liquid oxygen (LOX)/liquid hydrogen (LH2) used as the propellant. The fuel turbopump (FTP) and oxidizer turbopump (OTP) are driven by high-temperature hydrogen after cooling a combustion chamber. The pumps in the turbopumps feed LH2 or LOX by rotating each turbine. The RSR engine generated $40 \mathrm{kN}$ at $100 \%$ thrust at sea level. Chamber pressure and specific impulse were 3.4 MPa and

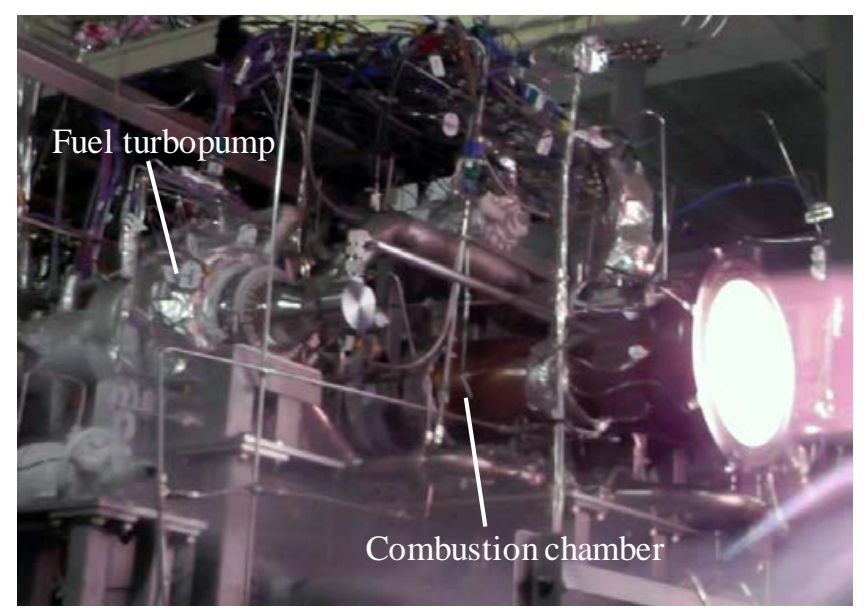

Figure 1. Static-firing test of RSR engine.
320 seconds at $100 \%$ thrust. This engine was designed for a launch vehicle capable of vertical takeoff and landing. Also, reignition and throttling from 40 to $100 \%$ are possible with the opening ratio of flow control valves changing. The staticfiring test was conducted over 50 times, and the engine was overhauled a few times during the test campaign.

Figure 3 is the time series of discharge pressure of an inducer and chill-down valve signal. The $\mathrm{x}$-axis is transformed into a range between 0 and 100; 0 and 100 indicate the beginning and end of the sequence respectively. The $y$-axis is normalized by the min-max normalization. The $\mathrm{x}$-axes and $\mathrm{y}-$ axes of all the graphs shown below are transformed by the same way in this paper. The sequence in this case consists of five combustion phases and four chill-down phases. Various sequences were conducted in the test campaign, but this study applies the sequence the test campaign conducted the most often. Figure 4 is a schematic of LH2 flow around the pump. The pump consists of a two-stage centrifugal pump with an inducer and connects with a turbine along an axis, which is a rotating shaft. The red circle marked "Res1" in Fig. 4 indicates the pressure sensor that measures the inducer discharge pressure shown in Fig. 3.

During the combustion phase, LH2 is fed by driving the FTP. Since the main valve opens, the chill-down valve and the bearing chill-down valve close, LH2 is circulated through the bearings by the FTP (Fig. 4 (a)). LH2 flowing through the bearing between the 2nd-stage impeller and the turbine returns to the bearing between the 1st-stage and 2nd-stage impellers through the inside of the hollow shaft. On the other hand, during the chill-down phase, the propellant is moved by only tank pressure because the FTP does not operate. Since the main valve closes, the chill-down valve repeatedly opens and closes, and the bearing chill-down valve opens after engine cutoff, then LH2 flows to cool the pump and bearings (Fig. 4 (b)). Opening and closing the chill-down

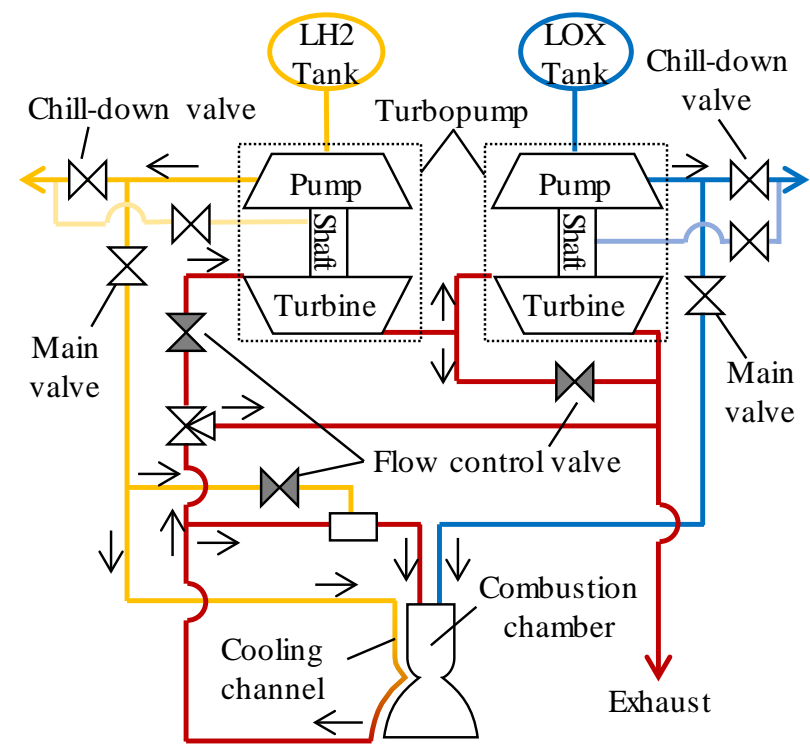

Figure 2. System diagram of RSR engine. 
valve repeatedly agitates the LH2 in the FTP and cools the pump and bearings effectively. As shown in Fig. 4, the stream of LH2 differs between the combustion and chill-down phases and is greatly affected by the valves.

\subsection{Sensors}

Engine performance in the static-firing test campaign was measured by over 350 sensors including measurements of ground equipment. This study used 60 sensors mounted on the engine: 26 pressure sensors, 26 thermocouples, four flowmeters, two rotation speed sensors, and two axial displacement sensors for the turbopumps. The system diagram in Fig. 2 indicates the main pipes of the RSR engine. The SLS can simulate the behavior of 30 sensors mounted on these pipes. The sensors that can be modeled by the SLS are listed in Table 1 . The remaining 30 sensors, mostly on the
A: Combustion
- Outlet pressure of inducer

B: Chill-down ----. Signal of chill-down valve

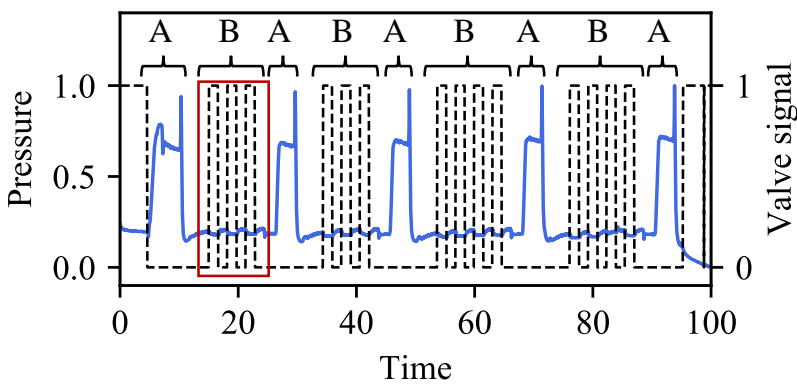

(a) Whole sequence turbopumps measured pressure and temperature around the bearings.

The bearings can rotate smoothly while the liquid propellant flows around them. If the propellant is vaporized because of some problem during turbopump operation, the bearings do not work, and severe damage to the turbopumps will result. Res2 and Res3 in Fig. 4 are the thermocouples for monitoring this. Res2 measures the temperature on the outside surface of the bearings, and Res3 measures the temperature of LH2 flowing around the bearings. The developed SLS model cannot simulate the flow indicated by blue arrows in Fig. 4 because flow channels are complicated and, instead, regards the area enclosed by a green box as one pump component. Res1, which measures the discharge pressure of an inducer, is included in the component. Therefore, Res1, Res2, and Res3 cannot be simulated by the SLS model.

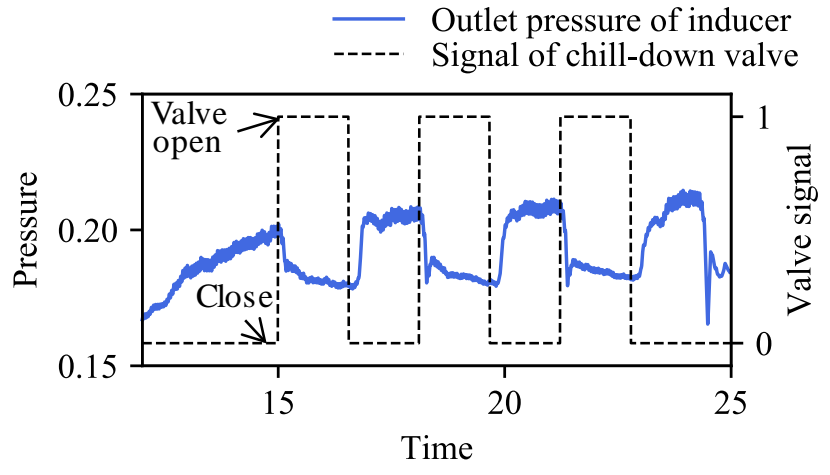

(b) Enlarged view of chill-down phase

Figure 3. Time series of discharge pressure of inducer and chill-down valve signal.

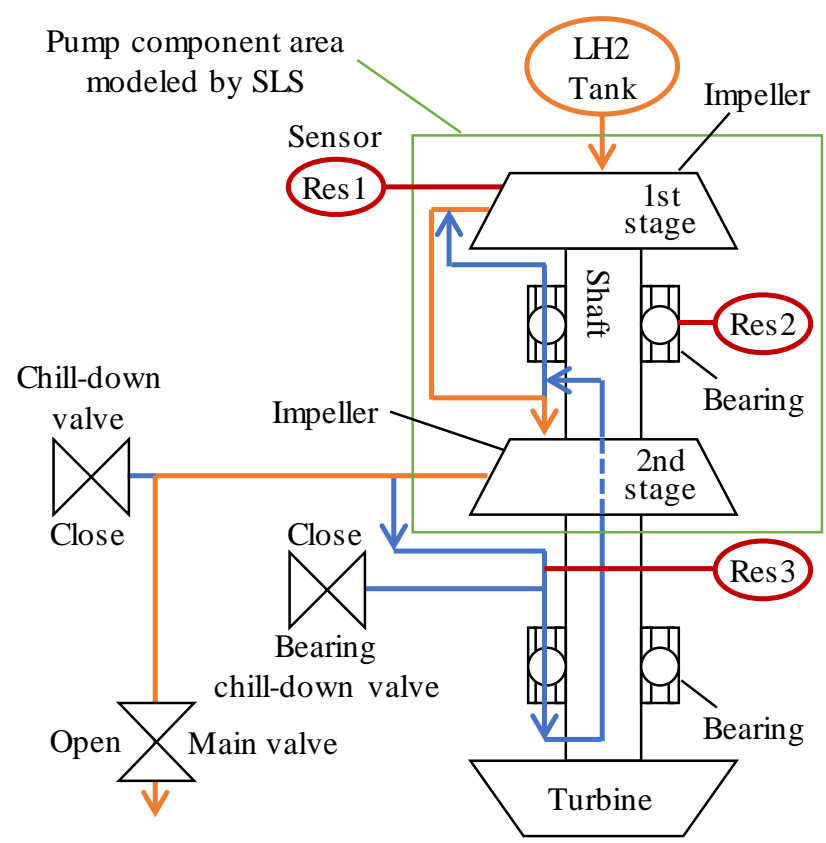

(a) Flow of LH2 during combustion phase

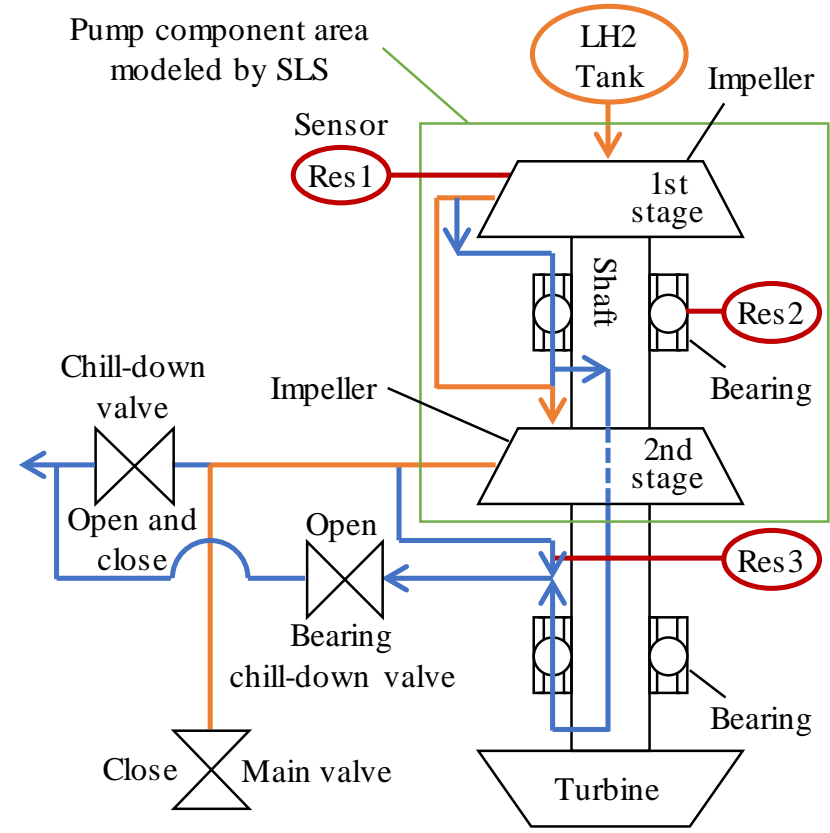

(b) Flow of LH2 during chill-down phase

Figure 4. Schematic of LH2 flow around pump. 
Table 1. Sensors that can be modeled by SLS.

\begin{tabular}{|l|l|l|l|}
\hline Number & Sensor & Number & Sensor \\
\hline Exp1 & Combustion pressure & Exp16 & Volumetric flow rate downstream of OTP \\
\hline Exp2 & Pump discharge pressure of FTP & Exp17 & Rotation speed of FTP \\
\hline Exp3 & Pump discharge pressure of OTP & Exp18 & Rotation speed of OTP \\
\hline Exp4 & Pressure of mixer at low temperature side & Exp19 & Pump discharge temperature of FTP \\
\hline Exp5 & Pressure of mixer at high temperature side & Exp20 & Pump discharge temperature of OTP \\
\hline Exp6 & Inlet pressure of regenerative cooling & Exp21 & Inlet temperature of regenerative cooling \\
\hline Exp7 & Outlet pressure of regenerative cooling & Exp22 & Outlet temperature of regenerative cooling \\
\hline Exp8 & Turbine inlet pressure of FTP & Exp23 & Turbine inlet temperature of FTP \\
\hline Exp9 & Turbine outlet pressure of FTP & Exp24 & Turbine outlet temperature of FTP \\
\hline Exp10 & Turbine inlet pressure of OTP & Exp25 & Turbine inlet temperature of OTP \\
\hline Exp11 & Turbine outlet pressure of OTP & Exp26 & Turbine outlet temperature of OTP \\
\hline Exp12 & Pressure of oxidizer side injector & Exp27 & Temperature of fuel side injector \\
\hline Exp13 & Pressure of fuel side injector & Exp28 & Temperature of oxidizer side injector \\
\hline Exp14 & Volumetric flow rate upstream of FTP & Exp29 & Temperature of lower chill-down valve for LH2 \\
\hline Exp15 & Volumetric flow rate downstream of FTP & Exp30 & Temperature of lower chill-down valve for LOX \\
\hline
\end{tabular}

\section{Creation of the Dataset}

\subsection{Procedure}

Figure 5 is an overview of the procedure for creating the dataset. The first step divides the static-firing test results into results from sensors that can be modeled by the SLS $\boldsymbol{X}=$ $\left(\boldsymbol{x}_{1}, \cdots, \boldsymbol{x}_{N x}\right)$ and from those that cannot $\boldsymbol{Y}=\left(\boldsymbol{y}_{1}, \cdots, \boldsymbol{y}_{N y}\right)$. $\boldsymbol{x}$ and $\boldsymbol{y}$ are the time series data of the sensors, and $\boldsymbol{x}_{i}=$ $\left(x_{i}^{1}, \cdots, x_{i}^{T}\right)^{t}$ and $\boldsymbol{y}_{j}=\left(y_{j}^{1}, \cdots, y_{j}^{T}\right)^{t}$, respectively. $x, y$ are instantaneous values of the time series data, and subscript $i \in$ $[1, N x], j \in[1, N y]$, and superscript $t \in[1, T]$ are the number of $\boldsymbol{X}=\mathbb{R}^{T \times N x}, \boldsymbol{Y}=\mathbb{R}^{T \times N y}$, and time, respectively. To train the regression model, $\boldsymbol{X}$ and $\boldsymbol{Y}$ are used as the explanatory variable and response variable. Then, $M$ explanatory variables $\left(\widetilde{\boldsymbol{X}}^{1}, \cdots, \widetilde{\boldsymbol{X}}^{M}\right)$ are created by the developed SLS model $g(\boldsymbol{\theta})$ with $\boldsymbol{\Theta}=\left(\boldsymbol{\theta}^{1}, \cdots, \boldsymbol{\theta}^{M}\right)$. $\boldsymbol{\Theta}$ is created by the Monte-Carlo method based on model parameter $\boldsymbol{\theta}$, fitted to the static-firing test results with data assimilation (Sato et al., 2019). $M$ response variables $\left(\widetilde{\boldsymbol{Y}}^{1}, \cdots, \widetilde{\boldsymbol{Y}}^{M}\right)$ are obtained by the trained regression model $f(\boldsymbol{X})$. Finally, by combining $\left(\widetilde{\boldsymbol{X}}^{1}, \cdots, \widetilde{\boldsymbol{X}}^{M}\right)$ and $\left(\widetilde{\boldsymbol{Y}}^{1}, \cdots, \widetilde{\boldsymbol{Y}}^{M}\right)$, the dataset $\mathcal{D}=\left(\left(\widetilde{\boldsymbol{X}}^{1}, \widetilde{\boldsymbol{Y}}^{1}\right), \cdots,\left(\widetilde{\boldsymbol{X}}^{M}, \widetilde{\boldsymbol{Y}}^{M}\right)\right)$ is obtained.

This study uses 24 cases out of over 50 cases of static-firing test results to train the regression model, and the gross number of $T$ is approximately 650,000. The test data for the regression model is the time series shown in Fig. 3, and its $T$ is approximately $30,000 . N x$ is 41 consisting of the 30 sensors listed in Table 1 and signals of valves, and $N y$ has three members: Res1, Res2, and Res3 shown in Fig. 4.

\subsection{Regression Model}

The sequence in Fig. 3 is strongly affected by the valve behavior. The flow direction around the pump in Fig. 4 is different between the combustion and chill-down phases as well. Res2 in Fig. 4 measures the temperature of the outside surface of the bearing, not the fluid temperature. Res3 measures the fluid temperature after it flows through the impellers and complicated channels, and it is affected by heat transfer from thermal mass especially during the chill-down phase. This study mainly aims to investigate whether the regression model can complement the dataset using a simple linear regression model, Ridge regression, but plans on using a nonlinear regression model to improve the prediction accuracy further.

\subsection{Clustering for Time-Varying Operating Conditions}

The main purpose of the static-firing test campaign was to determine an operating point that would allow the engine to operate normally. Therefore, this increases the amount of data for the combustion phase. Even if a nonlinear regression model is used, the usual regression models tend to value the major phase having the most data. Although a minor phase with a limited amount of data tends to be neglected, it is ideal for practicing fault detection and diagnosis for sequences that include both the major and minor phases. To prevent any bias caused by a difference in the amounts of data and the physical phenomena shown in Fig. 4, $\boldsymbol{X}$ is divided into clusters in advance, and regression models are individually trained on each clustered $\boldsymbol{X}$. The procedure for a training regression model is shown in Fig. 6.

Initially, the static-firing test results are divided into training and test data, and $\boldsymbol{X}$ of the training data is divided into $N c$ 
clusters. This study uses the Gaussian Mixture Model (GMM) as a clustering method as follows:

$$
\begin{aligned}
& p(x)=\sum_{c=1}^{N c} \pi_{c} \mathcal{N}\left(x \mid \boldsymbol{\mu}_{c}, \boldsymbol{\Sigma}_{c}\right) \\
& \sum_{c=1}^{N c} \pi_{c}=1
\end{aligned}
$$

where, $N c$ is 20 and $c \in[1, N c]$. The number of supposed phases according to operating conditions in the training data was around 20, so this study uses only $N c=20$. Mixture weight $\pi_{c}$, mean vector $\boldsymbol{\mu}_{c}$, and covariance matrix $\boldsymbol{\Sigma}_{\mathrm{c}}$ are obtained by the EM algorithm.

Then, $\boldsymbol{Y}$ is also divided into $N c$ clusters by label $\boldsymbol{L}=$ $\left(l_{1}, \cdots, l_{T}\right)^{t}$ from the GMM, and the regression models are trained from the thus obtained clusters. The regularization parameter of the Ridge regression model is obtained when the $\mathrm{R} 2$ score is maximum in 5-fold cross validation. $\widetilde{\boldsymbol{Y}}_{c}$ is calculated by inputting $\boldsymbol{X}_{c}$ divided by the GMM into the regression models trained on each cluster. Finally, the performance of the clustered regression models is evaluated by comparing the merged $\widetilde{\boldsymbol{Y}}$ with $\boldsymbol{Y}$ of the test data and evaluates it with the Root Mean Square Error (RMSE) as follows:

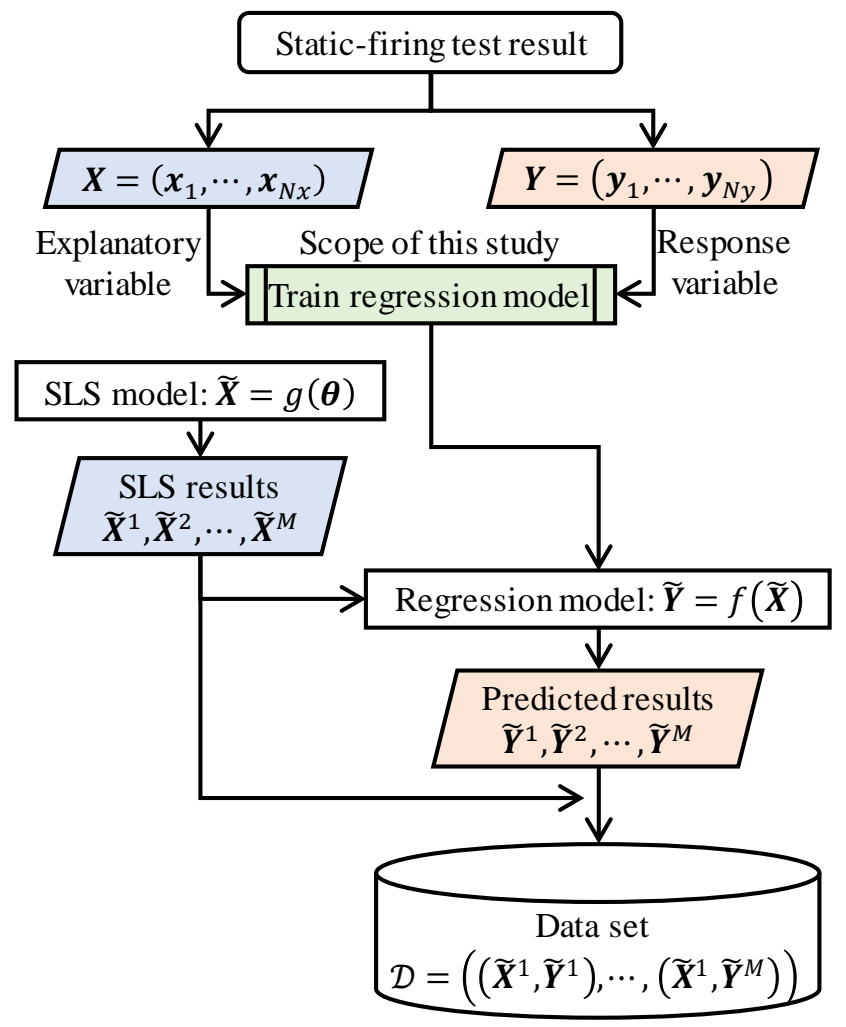

Figure 5. Overview of procedure for creating dataset.

$$
R M S E=\sqrt{\frac{\sum(\boldsymbol{y}-\tilde{\boldsymbol{y}})^{2}}{n}}
$$

where $n$ is the number of data.

\section{Prediction Results And Discussion}

\subsection{Prediction without Clustering}

Figure 7 is the comparison between the static-firing test results and predictions of the Ridge regressor without clustering $\boldsymbol{X}$ of the test data. The prediction of Res1 shown in Fig. 7 (a) generally agrees with the static-firing test results. Focusing on the area enclosed by a red box in Fig. 7 (a), however, the chill-down phase indicated by $\mathrm{B}$ and the

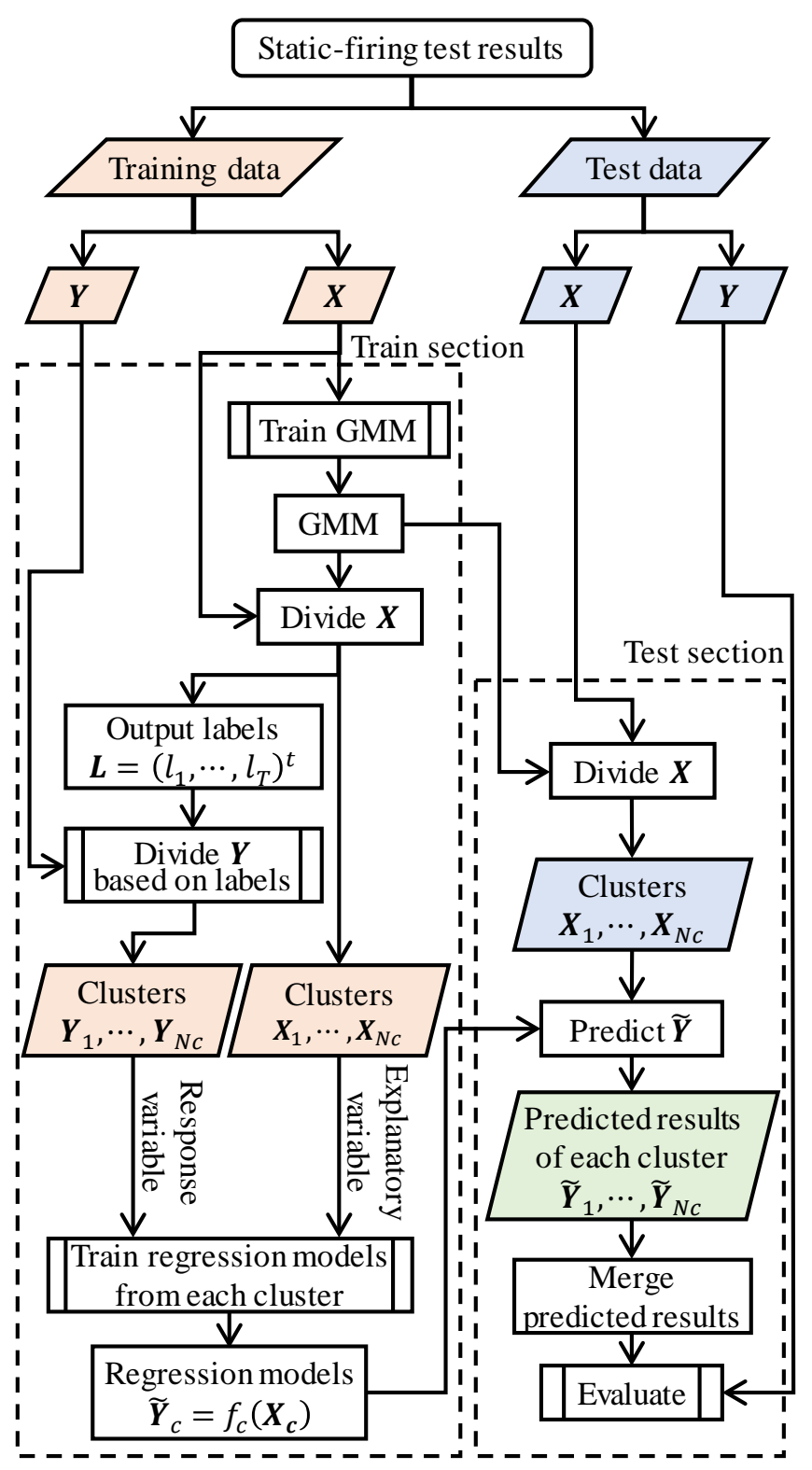

Figure 6. Procedure of training regression model 
switching point of the phases indicated by T1 and T2 are not predicted well, as can be seen from Fig. 7(b). Since Res1 (see Fig. 4) is the sensor that measures the inducer discharge pressure, its behavior is similar to the rotation speed, the discharge pressure of the turbopump, and flow rate during the combustion phase. The behavior of other pressure sensors is also similar, so the prediction accuracy for Res1 is improved because such sensors are used as the explanatory variables for the regression models. Moreover, since a large number of data for the combustion phase is included in the test data as well, the training regression model values this phase.

On the other hand, LH2 is fed by only tank pressure because the turbopump is stopped during the chill-down phase. The

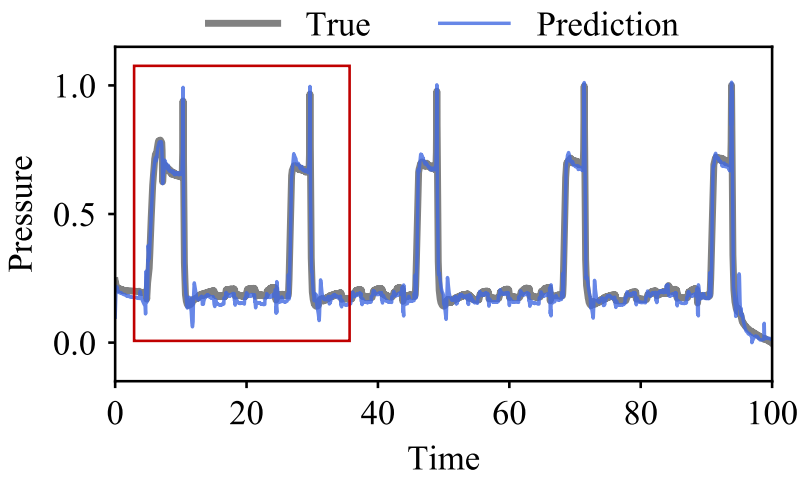

(a) Res1: inducer discharge pressure

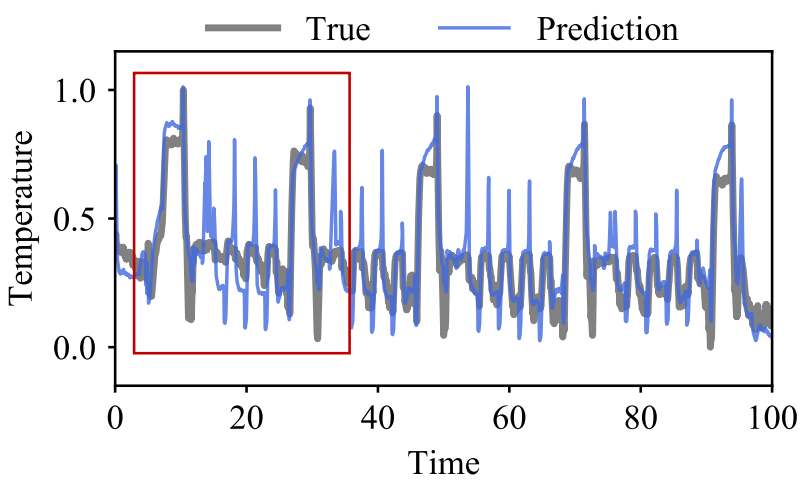

(c) Res2: bearing surface temperature

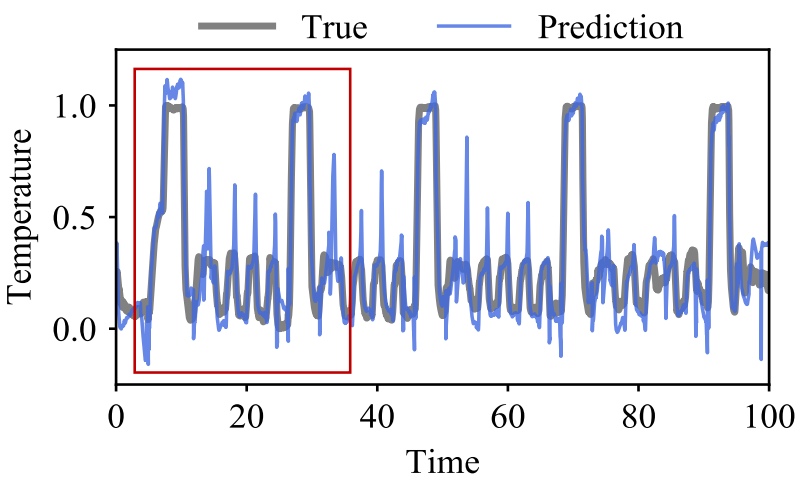

(e) Res3: LH2 temperature around bearing main valve closes, so its upstream fluid is strongly affected by the chill-down valve, but its downstream fluid is not affected. There are a few sensors whose behavior resembles that of Res1 during the chill-down phase. The number of data for the chill-down phase is smaller than that for the combustion phase, and the behaviors (such as valve operation) is different between the combustion and chilldown phases. The training regression model is inadequate for the above reasons.

Considering the prediction result for Res1, the regression model does not predict the behavior of the temperature Res2 and Res3 well. Some peaks are observed in the prediction results during the chill-down phase, so it is found that

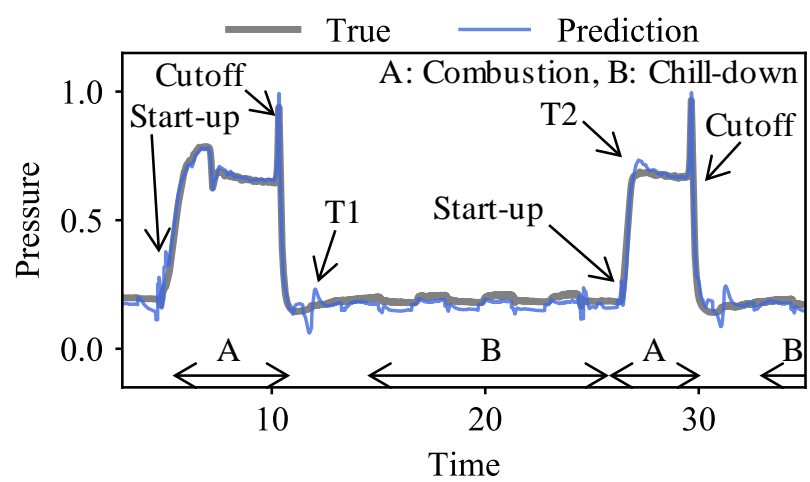

(b) Enlarged view at red box in Fig. 7 (a)

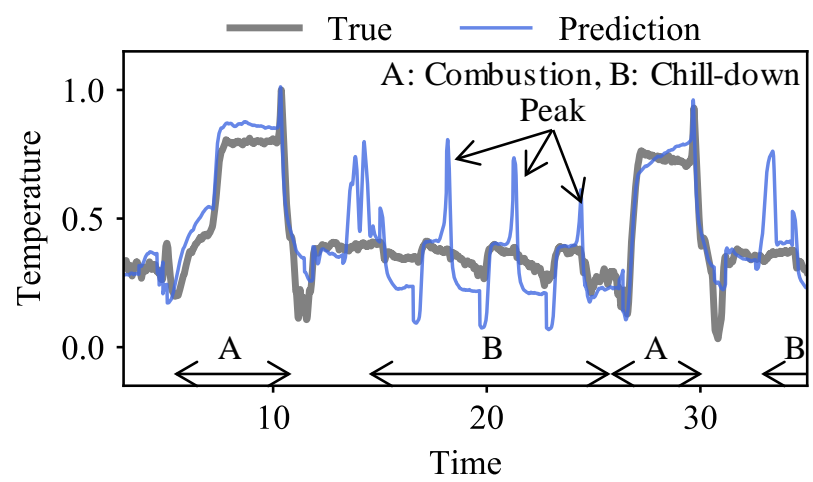

(d) Enlarged view at red box in Fig. 7 (c)

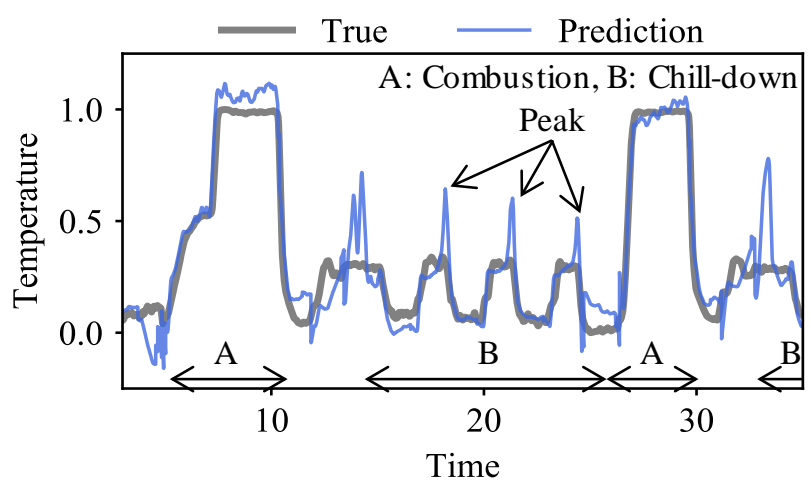

(f) Enlarged view at red box in Fig. 7 (e)

Figure 7. Comparison between static-firing test results and prediction of Ridge regressor without clustering. 
predicting the behavior of thermocouples is more difficult than the pressure sensor. When the turbopump is stopped and the propellant is stilled, the thermocouples are affected by heat from the outside and residual heat in the heat mass, such as pipes and pumps. The physical phenomena that affects the sensor behavior is the difference between the combustion and chill-down phases. Since the main valve closes during the chill-down phase, the explanatory variables for the behavior around the pump are limited. The chill-down phase behavior cannot be predicted by the regression model that values fitting the combustion phase.

\subsection{Prediction with Clustering}

Figure 8 is a comparison between the static-firing test results and prediction by the Ridge regressors with clustering $\boldsymbol{X}$ of the test data. The gray curves indicate the static-firing test result, and the colored curves indicate the prediction result. The color is changed for each cluster according to the legend at the top of Fig. 8 and common in Fig. 8. Note that clusters 1-4 expressed by the similar blue in Fig. 8 (b) are different. Although only four clusters are indicated here to distinguish the similar blue clusters, this sequence consists of more than four clusters as shown in Fig. 8. Focusing on the divided points of each cluster, the sequences are divided according to the steady state, transient state, and valve operation. The RMSE of the prediction results are listed in Table 2. All the response variables are improved by clustering.

Figure 8 (a) shows that the prediction result for Res1 agrees with the static-firing test result. As shown in Fig. 8 (b), an enlarged view of the area enclosed by the red box in Fig. 8 (a), the prediction accuracies for the switch of phases and the chill-down phase are improved. By individually training the regression models on each cluster, the minor chill-down phase had a small number of data and the major combustion phase had a large number of data, and were both valued in training the regression model. Switching the regression models predicts response variables well even if the physical phenomenon and the explanatory variable of each phase are different. As shown in Fig. 7 (b)-(f), the prediction results for Res2 and Res3 do not fit the static-firing test results even in the combustion phase, however, the accuracies of Res 2 and Res3 in Fig. 8 (b)-(f) are also improved by clustering. Focusing on the chill-down phase in Fig. 8 (d), the peaks shown in Fig. 7 (c) are prevented and the tendency of the prediction result is improved, but the prediction accuracy is worse than for Res1. On the other hand, the prediction result

Table 2. RMSE of prediction result.

\begin{tabular}{|c|c|c|}
\hline \multirow{2}{*}{ Sensor } & \multicolumn{2}{|c|}{ RMSE } \\
\cline { 2 - 3 } & without clustering & with clustering \\
\hline Res1 & 0.01320 & 0.003709 \\
\hline Res2 & 0.7764 & 0.4459 \\
\hline Res3 & 0.9447 & 0.4386 \\
\hline
\end{tabular}

for Res3 shown in Fig. 8 (e) generally fits the static-firing test result and it can predict not only the steady state during the combustion phase but also transient states, such as startup and cutoff. The chill-down phase is also predicted accurately, as shown in Fig. 8 (f). As mentioned in Section 2.2, the measurement point of Res3 differs from that of Res2. As shown in Fig. 4, Res2 measures the outside surface temperature of the bearing, which is changed by the heat transfer from LH2 and the bearing friction, not the fluid temperature directly, while Res3 measures the fluid temperature. The temperature change at Res 3 is based more on simple physical phenomena than is Res2, so the prediction accuracy for Res3 is better than Res2.

One method to improve the prediction accuracy for Res2 is to change the clustering method. Before training the regression model, $\boldsymbol{X}$ is divided into clusters by the GMM in order to reduce the bias that values a major phase with a large number of data. However, since the number of the clusters is a hyper-parameter of the GMM, there is the possibility that a minor phase with a small number of data could be classified in a neighboring cluster of a major phase, depending on the set cluster number. For the static-firing test result indicated by the gray curve in Fig. 8 (c), the temperature in the combustion and chill-down phases both gradually decrease. Although the tendency of the first chill-down phase particularly differs from the fourth, the GMM classifies them into the same cluster. Since the tendencies between phases in Res 1 and Res3 are almost the same, the prediction accuracy for Res2 seems to be improved by dividing the first chilldown phase as another cluster. Another method to improve the prediction accuracy for Res2 is to apply a nonlinear regression model. As mentioned above, since the Res2 is based on complicated physical phenomena, a linear regression model has trouble predicting such behavior. The linear regression model can predict the phase based on comparably simple physical phenomena, such as the combustion phase, so switching a linear and a nonlinear regression model according to each phase is effective in improving prediction accuracy.

\section{CONCLUSION}

For fault detection and diagnosis with machine learning, training data needs to be created for time-varying operating conditions. The authors have developed a system-level simulator that reasonably represents the global behavior of a reusable rocket engine. However, there are components that cannot be modeled by the system-level simulation, and they are also the targets of fault detection and diagnosis. The regression model was developed to complement the training data. Its explanatory variables are the sensors that can be modeled by the system-level simulation, and its response variables are the ones that cannot be modeled. The sequences of the reusable rocket engine consist of the various operating conditions, such as the combustion and chill-down phases. Training the regression model usually focuses on a major 
phase represented by a large amount of data, so a regression model based on the major phase tends to neglect a minor phase having a small number of data. Moreover, physical phenomena can change drastically between phases according to changes in the flow field inside pipes and turbopumps and in valve behavior. To solve such issues, the explanatory variables are divided into clusters by the Gaussian mixture model. The Ridge regression model trained on each cluster predicts the static-firing test results complied under various operating conditions well. From the above, the present method is shown to combine system-level simulation and the regression model used to generate training data for fault detection and diagnosis. However, the prediction accuracies for some sensor behavior are insufficient. Since nonlinear phenomena are dominant in a reusable rocket engine, improving prediction accuracy by applying a nonlinear regression model will be valuable work for the future.

\begin{tabular}{|l|l|l|l|l|l|l|l|l|l|l|l|l|l|l|l|l|l|l|l}
0 & 1 & 2 & 3 & 4 & 5 & 6 & 7 & 8 & 9 & 10 & 11 & 12 & 13 & 14 & 15 & 16 & 17 & 18 & 19 \\
\hline
\end{tabular}

Cluster number

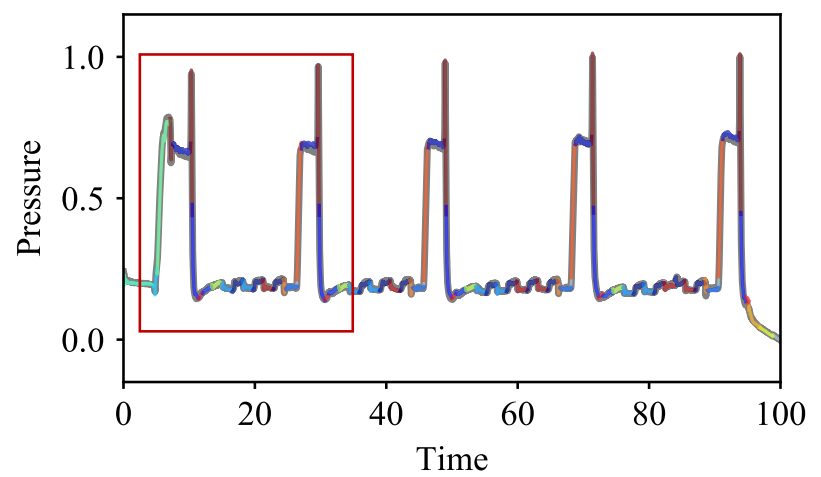

(a) Res1: inducer discharge pressure

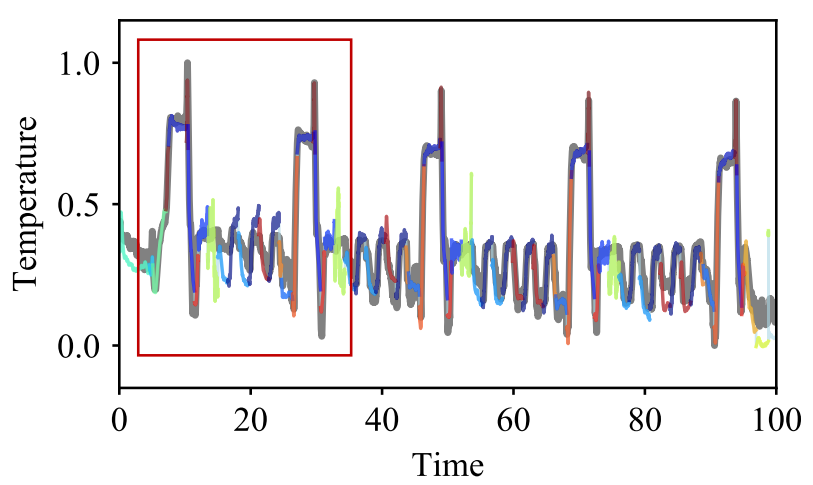

(c) Res2: bearing surface temperature

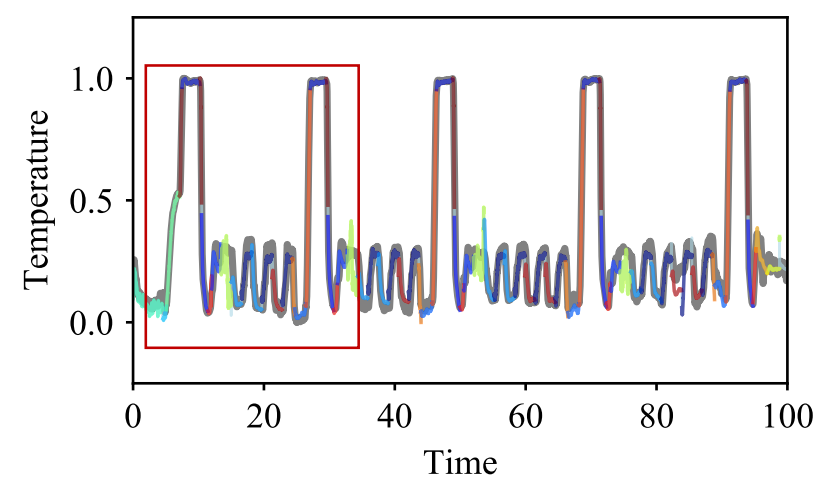

(e) Res3: LH2 temperature around bearing

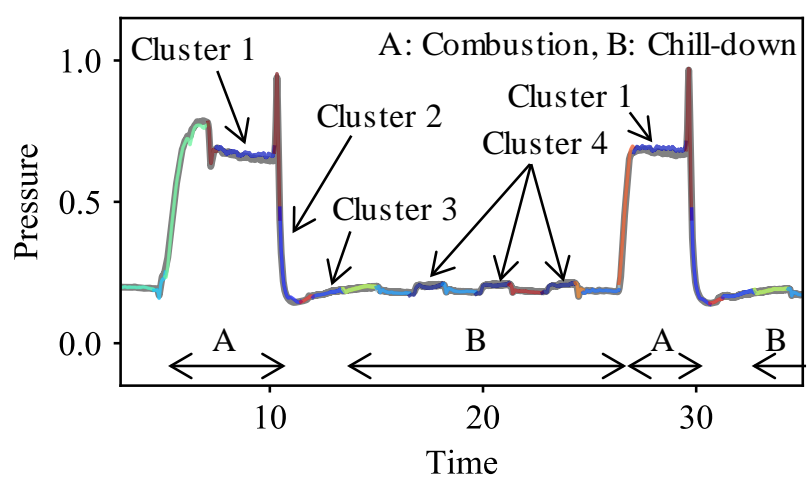

(b) Enlarged view at red box in Fig. 8 (a)

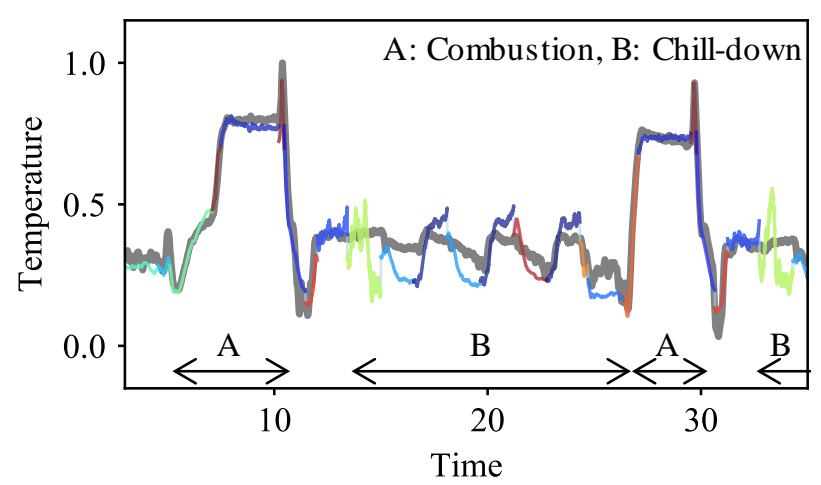

(d) Enlarged view at red box in Fig. 8 (c)

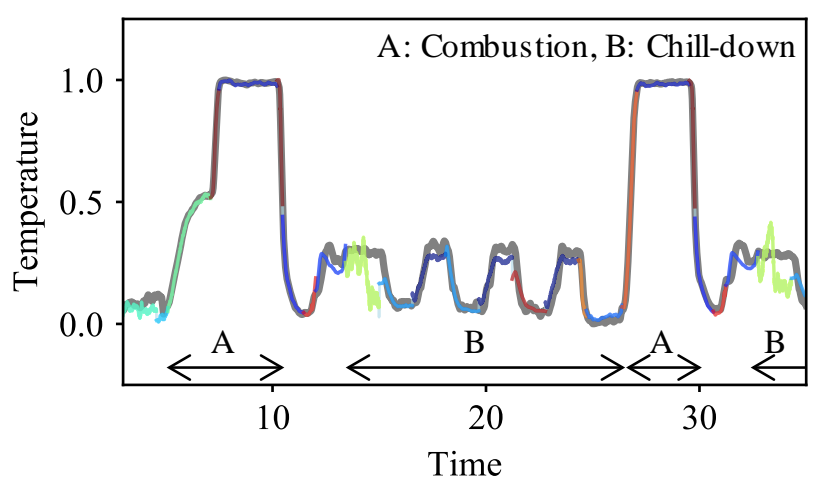

(f) Enlarged view at red box in Fig. 8 (e)

Figure 8. Comparison between test results and prediction of Ridge regressor with clustering. 


\section{REFERENCES}

Belapurkar, R., Yedavalli, R., \& Behbahani, A., (2011). Study of Model-based Fault Detection of Distributed Aircraft Engine Control Systems with Transmission Delays. 47th AIAA/ASME/SAE/ASEE Joint Propulsion Conference \& Exhibit, July 31-August 3, San Diego, CA. doi: 10.2514/6.2011-5795

Chowdhury, S. H., Ali, F., \& Jennions, I. K., (2019). A Methodology for the Experimental Validation of an Aircraft ECS Digital Twin Targeting System Level Diagnostics. Annual Conference of the PHM Society, vol. 11 (1). September 21-26, Scottsdale, AX. doi: 10.36001/phmconf.2019.v11i1.888

Frank, S., Heaney, M., Jin, X., Robertson, J., Cheung, H., Elmore, R., \& Henze, G., (2016). Hybrid Model-Based and Data-Driven Fault Detection and Diagnostics for Commercial Buildings: Preprint. 2016 ACEEE Summer Study on Energy Efficiency in Buildings, August 21-26, Pacific Grove, CA.

Kawatsu, K., (2019). PHM by Using Multi-Physics SystemLevel Modeling and Simulation for EMAs of Liquid Rocket Engine. Proceedings of IEEE Aerospace Conference. March 2-9, Big Sky, MO. doi: 10.1109/AERO.2019.8741827

Kimura, T., Hashimoto, T., Sato, M., Takada, S., Moriya, S., Yagishita, T., Naruo, Y., Ogawa, H., Ito, T., Obase, K., \& Ohmura, H., (2016). Reusable Rocket Engine: Firing Tests and Lifetime Analysis of Combustion Chamber. Journal of Propulsion and Power, vol. 32 (5), pp. 10871094. doi: 10.2514/1.B35973

Nonaka, S., Ogawa, H., Naruo, Y., \& Inatani, Y., (2011). System design and technical demonstrations for reusable sounding rocket. 20th ESA Symposium on European Rocket and Balloon Programmes and Related Research. May 22-26, Hyères, France.

Poon, J., Jain, P., Konstantakopoulos, C., Spanos, C., Panda, S. K., Sanders, S. R., (2017). Model-Based Fault Detection and Identification for Switching Power Converters. IEEE Transactions on Power Electronics, vol. 32 (2), pp. 1419-1430. doi: 10.1109/TPEL.2016.2541342

Sato, M., Hashimoto, T., Takada, S., Kimura, T., Onodera, T., Maruo, Y., Yagishita, T., Niu, K., Kaneko, T., \& Obase, K., (2014). Development of Main Propulsion System for Reusable Sounding Rocket. TRANSACTIONS OF THE JAPAN SOCIETY FOR AERONAUTICAL AND SPACE SCIENCES, AEROSPACE TECHNOLOGY JAPAN, vol. 12 (ists29), pp. Tm_1-Tm_6. doi: 10.2322/tastj.12.Tm_1

Sato, D., Tsutsumi, S., Hirabayashi, M., Kawatsu, K., \& Kimura, T., (2019). Estimation of Model Parameters in System-Level Simulation for Liquid Rocket Engines Using the Ensemble Kalman Filter. AIAA Propulsion and Energy 2019 Forum, August 19-22, Indianapolis, IN. doi: 10.2514/6.2019-4113
Sobie, C., Freitas, C., \& Nicolai, M., (2018). Simulationdriven machine learning: Bearing fault classification. Mechanical Systems and Signal Processing, vol. 99 (15), pp. 403-419. doi: 10.1016/j.ymssp.2017.06.025

Tsutsumi, S., Hirabayashi, M., Sato, D., Abe, M., Kawatsu, K., Sato, M., Kimura, T., \& Hashimoto, T., (2019). Fault Detection of a Reusable Rocket Engine using Phase Plane Trajectory Feature Vectors. Proceedings of the Annual Conference of the PHM Society, vol. 11 (1). September 21-26, Scottsdale, AX. doi: 10.36001/phmconf.2019.v11i1.771

Zolghadri, A., Leberre, H., Goupil, P., Gheorghe, A., Cieslak, J., \& Dayre, R., (2016). Parametric Approach to Fault Detection in Aircraft Control Surfaces. Journal of Aircraft, vol. 53 (3), pp. 846-855. doi: 10.2514/1.C032596 